\title{
fMRI Reveals a Common Neural Substrate of Illusory and Real Contours in V1 after Perceptual Learning
}

\author{
Marianne Maertens and Stefan Pollmann
}

\begin{abstract}
Perceptual learning involves the specific and relatively permanent modification of perception following a sensory experience. In psychophysical experiments, the specificity of the learning effects to the trained stimulus attributes (e.g., visual field position or stimulus orientation) is often attributed to assumed neural modifications at an early cortical site within the visual processing hierarchy. We directly investigated a neural correlate of perceptual learning in the primary visual cortex using fMRI. Twenty volunteers practiced a curvature discrimination on Kanizsa-type illusory contours in the MR scanner. Practice-induced changes in the BOLD response to illusory contours were compared between the pretraining and the
\end{abstract}

\section{INTRODUCTION}

Perceptual learning, the specific and relatively permanent modification of perception and behavior following sensory experience (Fahle \& Poggio, 2002), has been observed in a variety of tasks, including orientation discrimination (Fiorentini \& Berardi, 1980), texture discrimination (Karni \& Sagi, 1991), visual acuity (Fahle, Edelman, \& Poggio, 1995), discrimination of movement direction (Watanabe, Nanez, \& Sasaki, 2001), or visual search (Lobley \& Walsh, 1998). Within the psychophysics approach, the neural site of modification is inferred from the pattern of learning transfer. If learning effects are specific to the trained stimulus attributes, for instance, orientation or retinal position (Ahissar \& Hochstein, 1997), learning is assumed to occur in low-level visual areas, where neurons are orientation-selective and have small receptive fields (RFs). Therefore, training at one visual field position will alter neural activity only in the corresponding cluster of neurons, and leaving all others unaffected. In contrast, transfer of learning effects to untrained positions or orientations points to a level of modification higher up in the visual processing hierarchy. Here, neurons have larger RFs and are less sensitive to differences in stimulus position or orientation (Hochstein \& Ahissar, 2002). From the failure of learning transfer, however, it cannot necessarily be concluded

Otto-von-Guericke University, Magdeburg, Germany posttraining block in those areas of the primary visual cortex (V1) that, in the same session, had been identified to represent real contours at corresponding visual field locations. A retinotopically specific BOLD signal increase to illusory contours was observed as a consequence of the training, possibly signaling the formation of a contour representation, which is necessary for performing the curvature discrimination. The effects of perceptual training were maintained over a period of about 10 months, and they were specific to the trained visual field position. The behavioral specificity of the learning effects supports an involvement of V1 in perceptual learning, and not in unspecific attentional effects. that learning takes place at early processing levels. The learning-associated neural modifications might occur "centrally," and a lack of transfer then indicates that the central learning instance is required to interpret a new set of neural signals (Mollon \& Danilova, 1996). Consequently, we used functional magnetic resonance imaging (fMRI) to test the psychophysically derived hypothesis that perceptual learning is associated with neural modifications in early visual areas.

We trained our participants in the curvature discrimination of illusory contours (IC) in Kanizsa-type stimuli (Rubin, Nakayama, \& Shapley, 1997; Ringach \& Shapley, 1996). Their task was to decide whether the illusory contour was convex or concave (Figure 1). The percept of curvature was produced by creating a deviation in the angular section $\alpha$ in the inducing corner "pacmen" from $90^{\circ}$, resulting in concave figures for $\alpha$ smaller than $90^{\circ}$ and convex figures for $\alpha$ wider than $90^{\circ}$. The difficulty of the discrimination task was manipulated by varying the extent of the deviations from $90^{\circ}$ (Figure 2). Pre- and posttraining tests included hard cases only $\left(87^{\circ}<\alpha<\right.$ $\left.93^{\circ}\right)$, whereas during training, easier conditions $\left(87^{\circ} \geq\right.$ $\alpha \geq 93^{\circ}$ ) were used to optimize learning (Rubin et al., 1997). In contrast to earlier variants of the paradigm, which required subjective assessments of the clarity of contour impressions (Shipley \& Kellman, 1992), the curvature discrimination task provides a quantitative performance measurement for assessing training-related performance changes. 


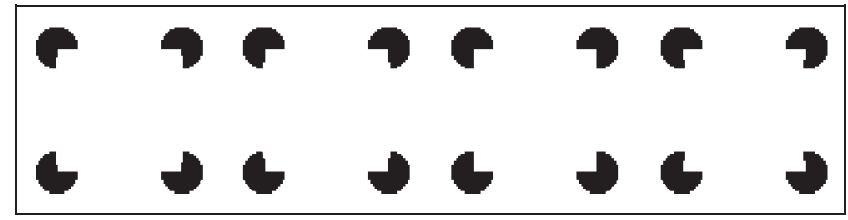

Figure 1. Sample stimuli. From left to right, extreme convexity passes into extreme concavity, the outermost stimuli are more extreme than the innermost and are classified as "fat" (left) and "thin" (right) more easily.

\section{Neurophysiology of Contour Completion}

The modifications to the neural circuitry involved in the task provide a model approach for neural recruitment processes, previously postulated as a candidate mechanism for perceptual learning (Gilbert, Sigman, \& Crist, 2001). The fundamental concept surrounding this approach is that in the absence of a physically defined curvature stimulus, contour completion processes are required to solve the curvature discrimination task. Kanizsa figures induce illusory contours which are collinear to the inducing edges. Completion processes of this type are assumed to be accomplished through an interaction between local cascade-like propagation of contour signals between neighboring neurons with small $\mathrm{RFs}$, and top-down influences from higher visual areas, which guide the cascade at earlier levels (Lee, 2003; Pillow \& Rubin, 2002). With the Kanizsa figure, some neurons are bottom-up stimulated by the luminancedefined edges of the inducers (Figure 3A, outermost neurons $\mathrm{O}$ and $\mathrm{O}$ ') that fall into their classic RFs. The cascade analogy demonstrates that these neurons activate their neighbors with a similar orientation preference and with RFs covering the intervening spatial positions between the inducers (Figure 3A, innermost neurons I and I'), an activation thought to occur via lateral horizontal connections (Angelucci et al., 2002; Bosking, Zhang, Schofield, \& Fitzpatrick, 1997; Toth, Rao, Kim, Somers, \& Sur, 1996; Gilbert \& Wiesel,

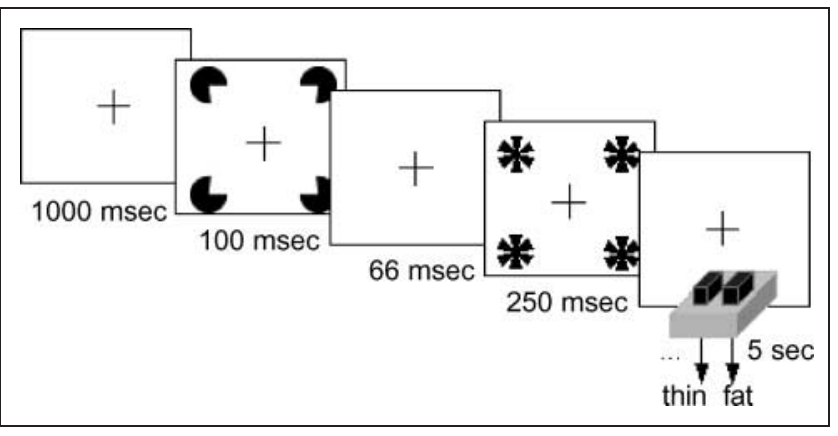

Figure 2. Illustration of the chronological sequence of events in a "thin" trial. Participants made an unspeeded 2-AFC response regarding the curvature of the stimulus.

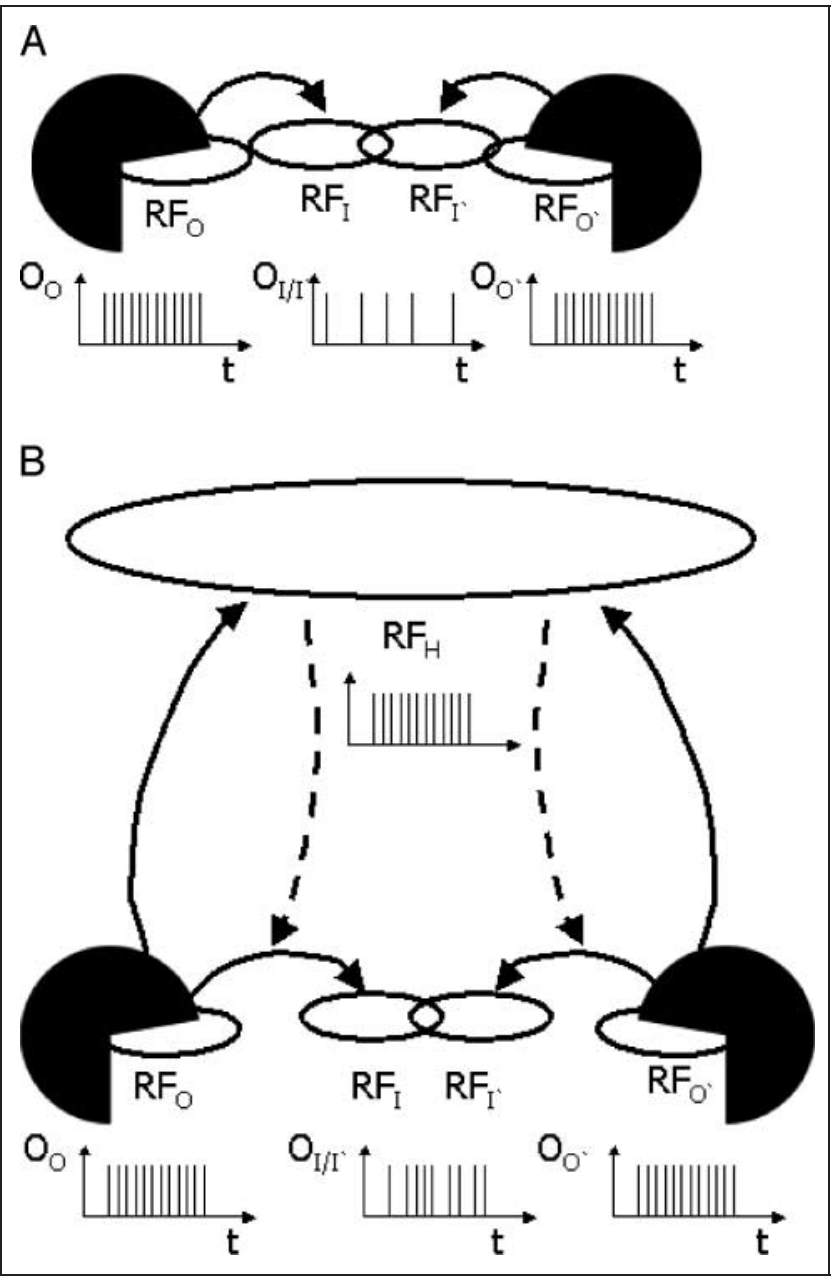

Figure 3. Illustration of the hypothetical mechanisms of contour completion. (A) Selective activity in neurons, which are stimulated by luminance-defined edges in their RF. The diagrams depict the idealized spiking activity $(\mathrm{O})$ over time $(t)$ for neurons that are either bottom-up-stimulated (neurons $\mathrm{O}$ and $\mathrm{O}^{\prime}$ ) or not (neurons I and $\mathrm{I}^{\prime}$ ). (B) In the course of training, activity is propagated via lateral and top-down connections to neurons with RFs "along" the illusory contour which have a collinear orientation preference, leading to an increase in their firing rate.

1992). In addition, the inducer-driven neurons are shown to propagate activity to neurons in higher visual areas with larger RFs (Figure 3B, neuron $\mathrm{H}$ ). These higher-level neurons may send feedback influences to inputting V1 neurons, possibly amplifying the input. These higher-level neurons may also direct the cascade signals by feeding into neurons with RFs positioned between the inducer-driven neurons.

The ease with which illusory contours are perceived depends critically on the support ratio (SR) of the stimulus ( $S R=$ the ratio of the luminance-defined part of the contour to its total side length). Increasing the interinducer distance makes the completion process more difficult, and at a certain distance, the task is no longer possible (Shipley \& Kellman, 1992). We propose that the cascade-like build-up of activity in V1 neurons is 
the neuronal mechanism that underlies this behavioral observation. A high SR allows for easy cascade build-up due to a strong signal being generated by the inducerdriven neurons and/or the need for a narrow gap being "bridged," difficult with low SR.

In the current experiment, an SR was employed, which led to chance performance in the beginning. Due to the large interinducer distance, the propagation of activity from inducer-driven to intervening neurons may initially fail to establish a continuous contour representation. Repeated exposure to the stimulus during training, however, may promote the propagation of activity from inducer-driven neurons to intervening contour-coding neurons via iterative interactions between lateral activation cascades and feedback signals. Because there is a positive indirect relationship between neural activity and the BOLD signal measured with fMRI (see Heeger \& Ress, 2002, for a review), we predict that the recruitment of interconnected neurons, representing the continuous illusory contour after learning, will lead to an increased BOLD response in the representation area of the illusory contour.

\section{Site of Learning}

Single-cell recording studies yielded evidence for ICrelated neural activity in the primary visual cortex (V1) (Lee \& Ngyuen, 2001; Ramsden, Hung, \& Roe, 2001; Sheth, Sharma, Rao, \& Sur, 1996; Grosof , Shapley, \& Hawken, 1993), as well as in peristriate area (V2) (von der Heydt, Peterhans, \& Baumgartner, 1984). There is still a debate, however, as to whether illusory contour processing involves computations as far down in the visual hierarchy as V1.

Functional imaging studies have revealed IC-related activity in V1 and/or V2 (Ritzl et al., 2003; Larsson et al., 1999; Ffytche \& Zeki, 1996; Hirsch et al., 1995) as well as in higher order visual areas such as the lateral occipital complex (LOC; Mendola, Dale, Fischl, Liu, \& Tootell, 1999). Stanley and Rubin (2003) distinguish between neural activity in response to an illusory contour and neural activity in response to an illusory figure. They suggest that illusory figure-related activity in the LOC is important to define a "salient region." For our stimuli, the bridging of a large interinducer gap $\left(11^{\circ}\right.$ visual angle) and a high precision curvature discrimination are two requirements that must be met simultaneously. Because the RF size of LOC neurons (e.g., Kastner et al., 2001) does not provide sufficient spatial resolution to discriminate the differences in curvature in the present task, we expected discrimination performance to rely on neurons in V1 where RF size is more appropriate (Hubel \& Wiesel, 1977). Activity in LOC neurons, however, may be necessary in the course of learning as the generator of feedback signals used to guide the completion mechanism in earlier visual areas. These predictions are in accordance with the "reverse hierarchy" model of per- ceptual learning (Ahissar \& Hochstein, 2004). Here, learning is proposed to be a top-down guided process beginning at high-level areas of the visual system and progressing backwards toward the input levels, which possess a better signal-to-noise ratio. Whereas the recruitment of IC-representing neurons should lead to an increase in BOLD response in the early visual cortex, based from the above, we would expect a decrease in BOLD signal due to decreasing activity in the LOC with increasing task proficiency.

\section{RESULTS}

\section{Psychophysical Performance}

Performance was measured using the magnitude of $\alpha$ required to produce reliable discrimination performance. For the test blocks, individual $80 \%$ performance thresholds were determined by fitting psychometric functions (Equation 1) to the data using Psignifit ${ }^{\circledR}$ version 2.5.41 (see http://bootstrap-software.org/psignifit/), a software package which implements the maximum likelihood method (Wichmann \& Hill, 2001a)

$$
\Psi(x)=\gamma+(1-\gamma-\lambda) \cdot F(x, \alpha, \beta)
$$

Being that psychometric functions of various types are easily convertible (Strasburger, 2001), we were able to choose the logistic function because of the straightforward interpretability of its function parameters (see Methods):

$$
F(x, \alpha, \beta)=\frac{1}{1+e^{-\frac{x-\alpha}{\beta}}}
$$

Participants were classified as learners or nonlearners on the basis of their performance patterns. The learner group was composed of seven subjects whose estimated posttraining thresholds were reduced by more than two standard deviations $(S D)$ of the pretraining thresholds and two subjects whose posttraining thresholds were reduced by more than $1 S D$. One additional participant was included because her discrimination threshold was reduced from pretraining $\left(4.0^{\circ}\right)$ to posttraining $\left(2.9^{\circ}\right)$, albeit the latter narrowly missed the limit of $1 S D\left(1.2^{\circ}\right)$ from pretraining threshold $\left(>2.8^{\circ}\right)$. Ten subjects were assigned to the nonlearning group due to their demonstration of chance performance throughout both test blocks, indicated by extreme threshold estimations $\left(\mathrm{th}_{\text {pretest }}=51.6^{\circ} ;\right.$ th $_{\text {posttest }}=33.8^{\circ} ;$ Figure 4$)$. Three of the remaining four subjects were not considered in the analyses due to ceiling effects. Although the fourth subject did improve significantly, his posttraining threshold was still higher than most learners' pretraining thresholds. Therefore, the (neural) processes involved 
Figure 4. Behavioral improvements during the learning experiment. The probability $(p)$ of "thin" responses (ordinate) is plotted as a function of the angular deviation from $90^{\circ}$ (abscissa, negative values indicate "fat" Kanizsa stimuli). The upper graphs depict performance in the pretraining block, the lower graphs performance in the posttraining block; the graphs on the left side belong to the learners, those on the right side to the nonlearners. Bars indicate bootstrapsampled standard errors of the mean (see Methods); interpolating lines represent the estimated psychometric functions, $\operatorname{th}(x)$ denotes the $80 \%$ performance threshold for condition $x$.

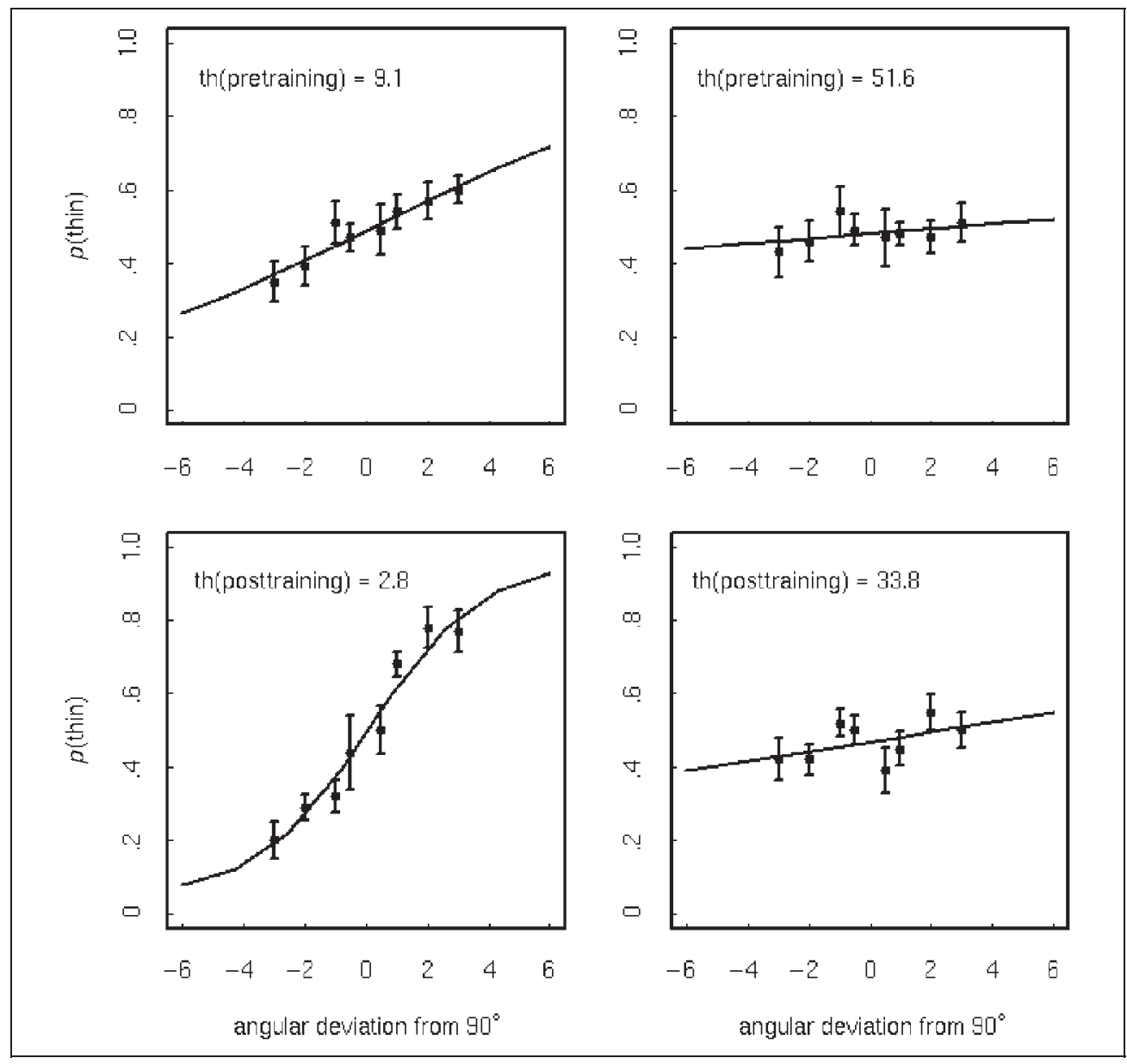

in his posttraining discrimination performance may have differed from those applied by the other subjects. As illustrated in Figure 4, the improvements in discrimination performance over the course of training were accompanied by a steepening of the slope of the psychometric function, approaching the ideal observer model of a step function in the second test block for the learners. The learner group as a whole showed a threshold reduction of $\alpha$, from $9.1^{\circ}$ to $2.8^{\circ}$, as a consequence of training. The posttraining threshold was observed to be smaller than the lower limit of the 95\% confidence interval around the pretraining threshold of $5.8^{\circ}$.

In the retest session, run approximately 10 months after the MR experiment, contour discrimination, at the old and at a new visual field position, was tested using equivalent threshold estimations as in the initial learning session. The learner group maintained its high performance level over time as indicated by an $80 \%$ retain threshold estimation of $\alpha=2.2^{\circ}$ at the old location that fell within the $95 \%$ confidence limits $\left[2.1^{\circ}, 3.6^{\circ}\right]$ around the posttraining threshold (Figure 5). In contrast, performance at the new location was significantly worse than at the old location. The learner's transfer threshold of $\alpha=7.1^{\circ}$ was outside the $95 \%$ confidence limits around the retain threshold $\left[1.5^{\circ}, 4.1^{\circ}\right]$.
The nonlearners showed some improvement from the posttraining to the retest session, as their retain threshold of $6.3^{\circ}$ was markedly (although not significantly) lower than their posttraining threshold (Figure 5). Their retain threshold, however, was significantly higher than that of the learners. The nonlearners' transfer threshold of $35.0^{\circ}$ was significantly worse than that for retain performance, for which $95 \%$ confidence limits of $3.3^{\circ}$ and $24.8^{\circ}$ were estimated.

\section{fMRI Results}

Illusory contour-related activity was measured at the V1 representation areas of real contours presented at the same visual field location as the Kanizsa side edges, which were individually identified in the localizer scan for each subject. Table 1 contains the Talairach and Tournoux (1988) coordinates of individual activation maxima in the localizer scan in response to contralaterally presented real contours for 18 participants. ${ }^{1}$ The coordinate means were shown not to differ between learners and nonlearners [left: $x=-8.4 \pm 4.9$, $y=-89.4 \pm 4.3, z=3.6 \pm 5.0$ and $x=-8.8 \pm 2.7$, $y=-84.1 \pm 4.4, z=5.3 \pm 4.5$, respectively; $t(3,14)=$ $0.432, p=.158$; right: $x=9.11 \pm 4.7, y=-86.5 \pm 4.4$, 
Figure 5. Behavioral performance in the retest session. Axis labels are identical to those in Figure 4 The upper two graphs depict performance in the retain block, those in the lower row performance in the transfer block. The graphs on the left side belong to the learner group, those on the right side to the nonlearners. Bars indicate bootstrap-sampled standard errors of the mean; interpolating lines represent the estimated psychometric functions, $\operatorname{th}(x)$ denotes the $80 \%$ performance threshold for condition $x$.

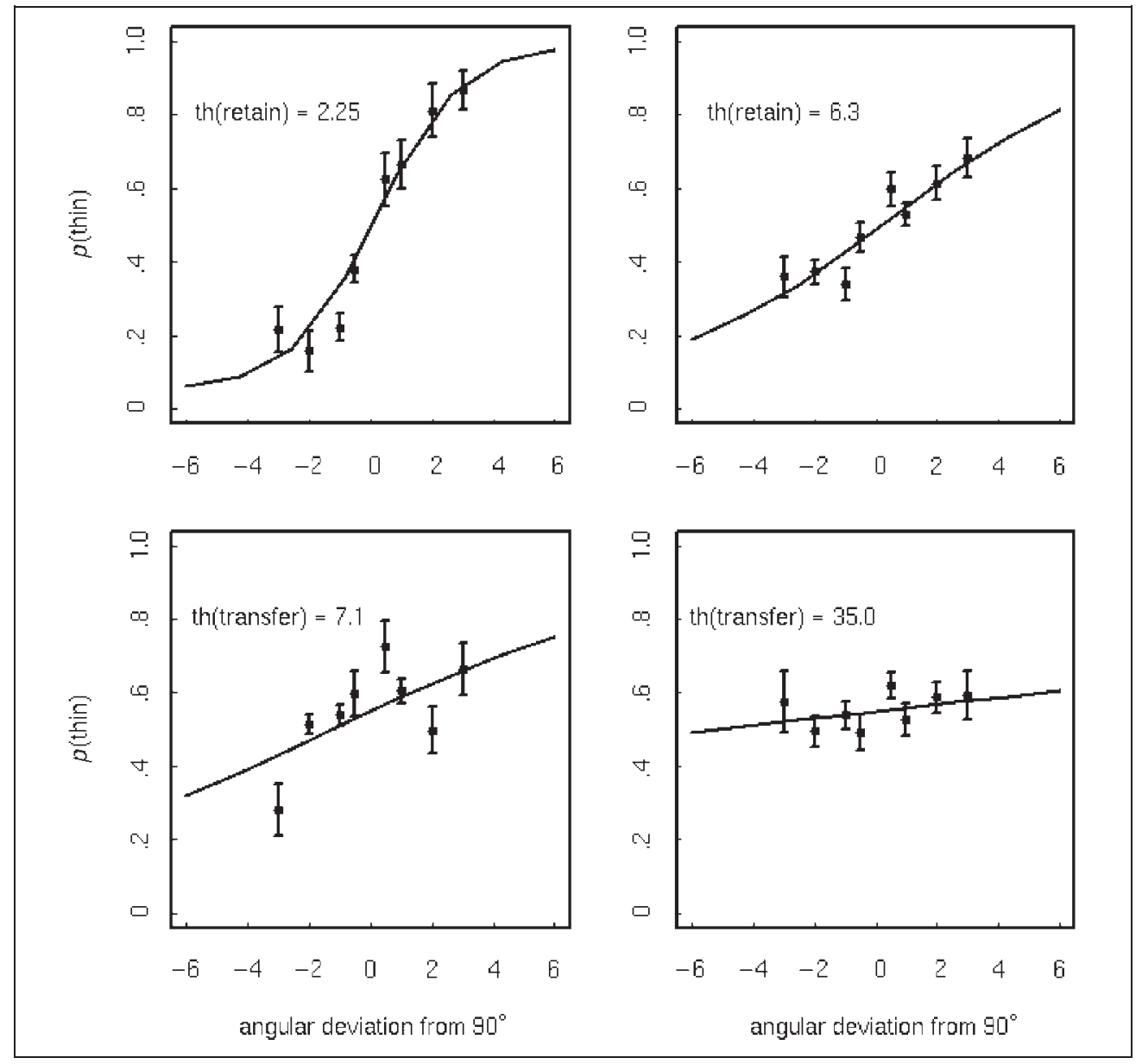

$z=5.2 \pm 6.0$ and $x=10.3 \pm 2.0, y=-83.7 \pm 4.0, z=7.6 \pm$ $5.1 ; t(3,15)=0.176, p=.474]$. Figure $6 \mathrm{~A}$ depicts the corresponding $\operatorname{SPM}\{Z\}$ for one subject illustrating that functional areas can be identified unambiguously with respect to anatomical and functional landmarks. The activation was required to lie at the banks of the calcarine fissure, typically in the vicinity of its fundus. Additionally, we were able to identify the activation elicited by vertical meridian stimulation, which indicates the border between V1 and V2 (see Methods for details).

For learners, significant differences in BOLD responses between the pre- and posttraining blocks were observed in the left hemisphere $[q(\mathrm{FDR}) \leq .05]$. There was a similar trend observed in the right visual cortex, but was shown to be insignificant after correction for the false discovery rate (FDR) (Figure 6B). For nonlearners, fMRI responses between pre- and posttraining blocks did not differ in either hemisphere $[q(\mathrm{FDR})>.05$; Figure 6B].

In addition to our hypothesis-driven investigation of V1 regions of interest (ROIs), we carried out a wholebrain analysis to search for higher-level areas potentially involved in perceptual learning of illusory contour discrimination. A $t$ test between subjects was performed to compare training-induced activation changes between the groups. Significant differences were observed bilaterally in the LOC (Grill-Spector et al., 1998; $x=-34, y=-66, z=-3, p=.0003 ; x=34, y=-63$, $z=2, p=.0004$; Figure 7 ), resulting from a differential effect within groups. Although the learning group exhibited a significant decrease of activation from the pre- to the posttraining block in these areas (left LOC: $p<.0002$; right LOC: $p<.005)$, no differences were observed for the nonlearners $(p>.05$ in the left and right LOC).

\section{DISCUSSION}

The central finding from this study is that perceptual learning of illusory contour discrimination was accompanied by an increase in neural activity in V1. At the same time, a decrease of activation over the course of learning was observed in the LOC. The signal increase in V1 was specific in several regards. First, it occurred at the retinotopic representation area of the illusory contour. Second, it occurred only in subjects who improved significantly in illusory contour discrimination over the test session and who, in addition, showed retinotopically specific improvements at the trained visual field locations at the 10-month follow-up. 
Table 1. Talairach Coordinates for Activation Peaks in Individual $\operatorname{SPM}\{Z\}$ (see Figure 4 for an example) of Increased Activation in Response to Contralateral Real Contours versus Rest in the Retinotopic Mapping Protocol

\begin{tabular}{|c|c|c|c|c|c|c|c|c|}
\hline \multicolumn{5}{|c|}{ Left Hemisphere } & \multicolumn{4}{|c|}{ Right Hemisphere } \\
\hline & $x$ & $y$ & $z$ & $\begin{array}{c}Z \\
\text { Value }\end{array}$ & $x$ & $y$ & $z$ & $\begin{array}{c}Z \\
\text { Value }\end{array}$ \\
\hline 1 & 1 & -86 & 5 & 2.8 & 13 & -80 & 5 & 2.8 \\
\hline 2 & -12 & -92 & 5 & 3.3 & 7 & -92 & 2 & 2.9 \\
\hline 3 & -12 & -91 & 12 & 2.1 & 16 & -88 & 13 & 2.7 \\
\hline 4 & -10 & -87 & 6 & 2.6 & 2 & -89 & 14 & 2.6 \\
\hline 5 & -7 & -90 & 1 & 2.5 & 10 & -84 & 8 & 2.4 \\
\hline 6 & - & - & - & - & 15 & -86 & 0 & 1.6 \\
\hline 7 & -8 & -86 & -3 & 3.1 & 13 & -83 & 6 & 3.0 \\
\hline $8^{\mathrm{a}}$ & -7 & -87 & 0 & 2.3 & 11 & -85 & 1 & 2.9 \\
\hline 9 & -10 & -80 & 5 & 3.4 & 10 & -80 & 8 & 4.6 \\
\hline 10 & -16 & -80 & 5 & 2.8 & 10 & -80 & 14 & 2.5 \\
\hline 11 & -8 & -89 & 7 & 2.6 & 9 & -83 & 12 & 2.7 \\
\hline 12 & -4 & -86 & 5 & 3.0 & 7 & -80 & 8 & 3.9 \\
\hline 13 & -7 & -77 & 12 & 2.6 & 7 & -77 & 11 & 2.9 \\
\hline 14 & -8 & -90 & 10 & 3.0 & 12 & -91 & 11 & 4.1 \\
\hline 15 & -7 & -81 & 6 & 2.5 & 10 & -86 & 0 & 2.0 \\
\hline 16 & -14 & -85 & -5 & 3.4 & 6 & -89 & -3 & 3.3 \\
\hline 17 & -8 & -84 & 3 & 2.5 & 8 & -87 & 11 & 3.0 \\
\hline 18 & -9 & -98 & 0 & 2.7 & 6 & -91 & 0 & 1.6 \\
\hline 19 & -9 & -87 & 8 & 3.1 & 13 & -85 & 2 & 2.2 \\
\hline
\end{tabular}

${ }^{\mathrm{a}}$ For Participant 8 , the anatomical sites of the activation changes are depicted in Figure 4 by overlaying the $\operatorname{SPM}\{Z\}$ onto the individual brain anatomy.

\section{Neural Site of Perceptual Learning}

In subjects who showed a significant improvement in illusory contour discrimination over the session, a BOLD signal increase was observed in the retinotopic representation area of the illusory contours (independently mapped with real contours at the same position), along the banks of the calcarine fissure, and within the bounds of the vertical meridian representation, which marks the borders between V1 and V2.

To our knowledge, this is the first retinotopic localization of perceptual learning-related signal changes in V1. In a past PET study investigating the neural correlates of perceptual learning in an orientation discrimination task (Schiltz et al., 1999), decreasing activity was reported in striate and extrastriate cortices. Unfortunately, as the authors remarked themselves, they were not able to decide whether the results could better be accounted for in terms of bottom-up or top-down effects. They interpreted the observed activity decrease as a remote effect of diminished attentional modulation, instead of modifications in the primary visual areas themselves, such as the reduction in the number of responsive neurons as a consequence of a sharpening of their orientation tuning curves.

Schwartz, Maquet, and Frith (2002) explored perceptual learning effects for texture discrimination with fMRI and observed a learning-associated increase of the BOLD response along the calcarine fissure. Their functional localization of learning-induced activity increases, however, was based on the graphical overlap of the group activation maxima between the experimental contrast and the localizer. This procedure may have potentially introduced inaccuracies due to neglecting interindividual differences in the (functional) anatomy of the primary visual cortex.

Here, we defined our ROIs exclusively on the basis of the localizer contrast, enabling us to show that the observed learning-dependent signal increases from preto posttraining did not only spatially overlap with the activation in the localizer scan, but were positioned at exactly the same sites. The retinotopic specificity of the learning-associated activation in V1 is important, because it was predicted on the basis of the neural mechanisms which we assume to form the basis for illusory contour representation in V1.

\section{Perceptual Learning versus Attention}

Before we discuss possible neural mechanisms of perceptual learning in V1, alternative explanations should be considered. One potential cause for increased activation in visual areas is attentional modulation, previously reported in a number of studies (Pollmann, Weidner, Müller, \& von Cramon, in press; Pollmann, Weidner, Müller, \& von Cramon, 2000; Brefczynski \& DeYoe, 1999; Gandhi, Heeger, \& Boynton, 1999; Martinez et al., 1999; Büchel et al., 1998; Kastner, De Weerd, Desimone, \& Ungerleider, 1998; Tootell et al., 1998; Beauchamp, Cox, \& De Yoe, 1997; O'Craven, Rosen, Kwong, \& Treisman, 1997). It has been shown to be most prominent in higher-tier visual areas, but has also been observed as early as V1 (Somers, Dale, Seiffert, \& Tootell, 1999) and even in the lateral geniculate nucleus (O'Connor, Fukui, Pinsk, \& Kastner, 2002). We think that attentional modulation is an unlikely cause of our V1 activation, for the following reasons: (1) It is easy to focus attention on the part of the visual field between the inducers, so that attentional modulation may be expected to lead to an immediate increase of activation at the beginning of the experiment instead of an increase occurring in the final block of the experiment, as we have observed. Furthermore, focusing of visuospatial attention is typically accompanied by increased activation in the frontal eye fields and along the intra- 
Figure 6. Learning effects in the primary visual cortex. (A) Example $\operatorname{SPM}\{Z\}$ of left and right checkerboard contours versus baseline in the localizer scan registered on the individual anatomy of one participant (see Table 1 for the exact Talairach coordinates). (B) Percent signal change in response to illusory contours during pre- and posttraining stimulus presentations at the retinotopic representation of real contours in the left (bottom left) and right calcarine fissure (bottom right). The asterisk indicates that, according to the FDR-corrected significance criterion, a significant difference between the pre- and the posttraining blocks was observed only in the left hemisphere of the learner's group. The left diagram depicts the signal changes in the left hemisphere and the right diagram the signal changes in the right hemisphere. Error bars represent jackknifed standard errors of the mean.

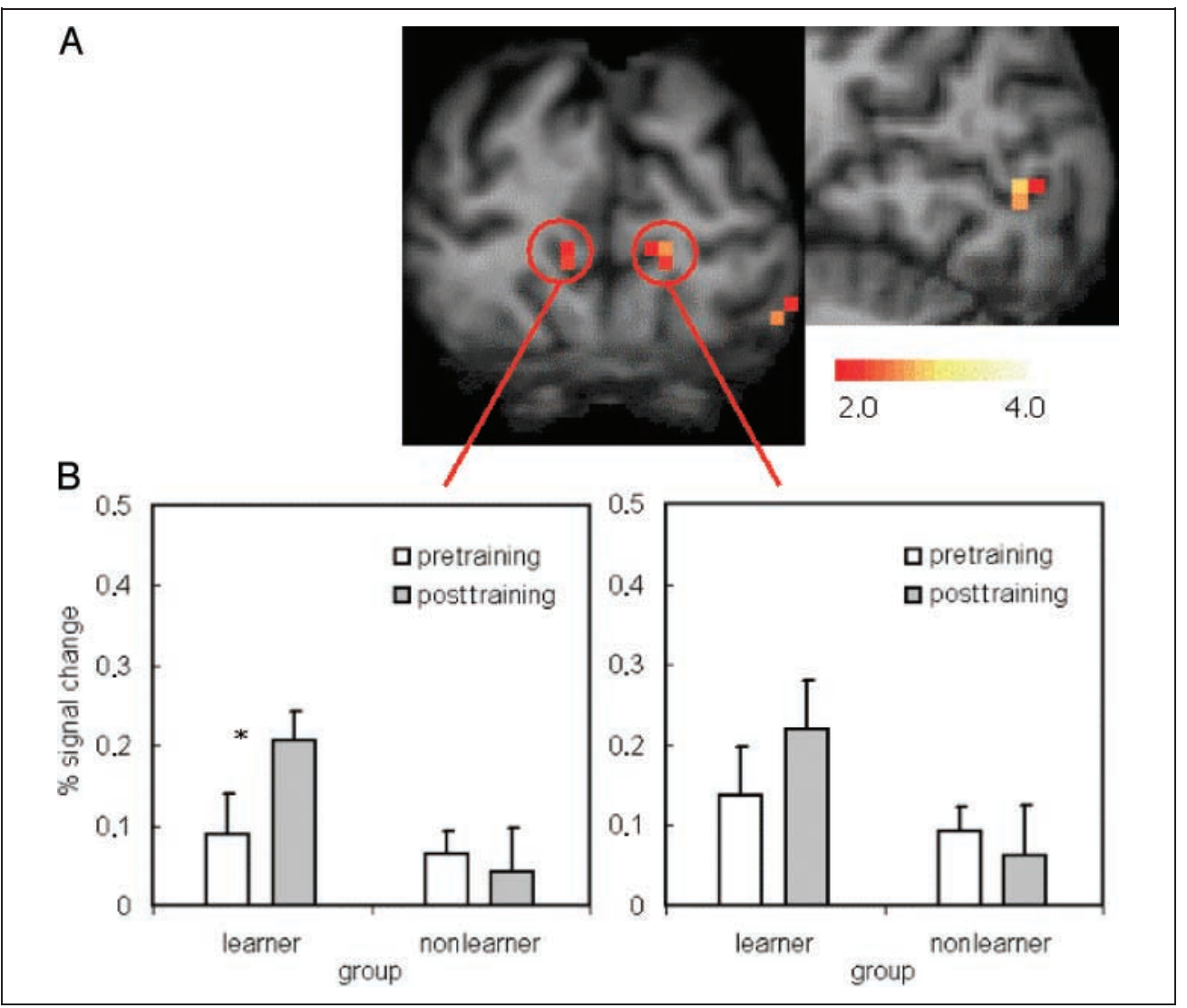

parietal sulcus. Although the signal increase in this "fronto-parietal network of attention" (e.g., Pollmann \& von Cramon, 2000; Gitelman et al., 1999; Corbetta et al., 1998) typically is much stronger than attentional modulation in V1, no such activation was observed in the present study. (2) The retinotopically specific increase of V1 activation was observed only in the subgroup of subjects who showed perceptual learning. Focused attention, however, may be especially beneficial

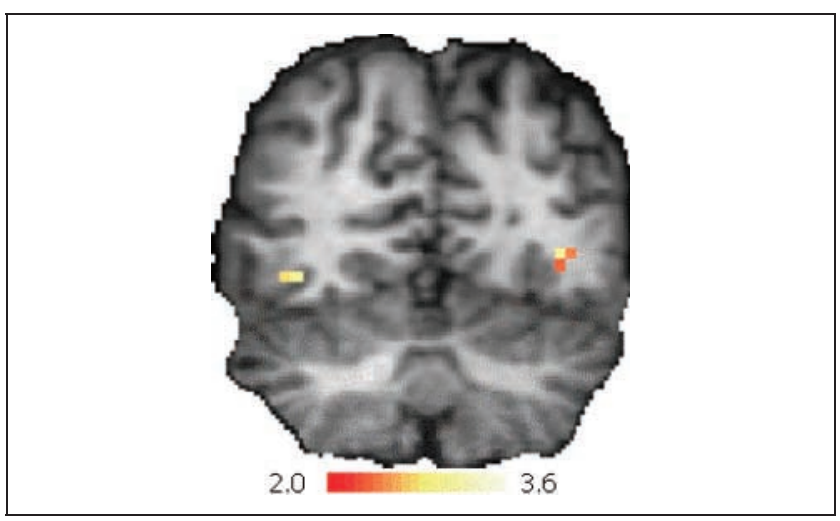

Figure 7. $\operatorname{SPM}\{Z\}$ indicating the degree of significance of the group difference for the contrast between pre- and posttraining BOLD responses. The map is overlaid on a coronal slice at $y=-66$ of an individual anatomical image of one of the participants. in the absence of perceptual learning. Analogous to the argument that attentional modulation may be most likely in the beginning of the experiment, when the discrimination performance is weak in all subjects, it may be postulated that towards the end of the session, attentional modulation may be strong in the nonlearners, and weak in the learners, which have an improved discrimination performance and less demand for attentional resources. This argument implies, however, that the improved contour discrimination among the "learners" indeed is caused by perceptual learning, and not by an improved ability to focus attention on the part of the visual field in which the inducers are presented. The data from our behavioral follow-up study speak against the latter alternative. If the improved discrimination performance in the learners was an attentional effect, there would be no reason to expect the retinotopic specificity of the discrimination improvement which we observed 10 months after the initial fMRI experiment. The follow-up data strongly suggest that improved performance was indeed due to lasting neuronal changes in a retinotopic visual area.

\section{Neural Mechanisms of Perceptual Learning}

Two components of learning in the curvature discrimination task can be distinguished: the formation of a 
contour representation over an extended area of space, and the tuning of neurons to detect small differences in curvature. The observed changes in the BOLD signal rather reflect the former component, the spread of activation from the inducer-representing neurons to neighboring neurons in the form of the cascade model, whereas the latter will probably lead to spatial changes of activation which are too subtle to be picked up by current fMRI techniques. The current results support the idea that contour integration relies on collinear completion mechanisms in the primary visual cortex (Lee \& Nguyen, 2001). It has been reported that longrange horizontal connections exhibit orientation-specific anisotropy as they preferentially link patches of neurons with collinear orientation preference and co-axially aligned RFs (Bosking et al., 1997) over cortical distances between 0.5 and $2 \mathrm{~mm}$ (Angelucci et al., 2002). Toth et al. (1996) demonstrated that long-range influences between neurons with collinear orientation preference are facilitative as long as the relative contrast of the surround was higher than that of the center stimulus. Because the illusory contours were not contrast-defined at all, neurons stimulated by the vertical edges of the pacmen should exert exclusively facilitating influences on neurons with intermediately adjoining iso-oriented RFs (Figure 3A). This is analogous to effects observed within the context of lesion-induced reorganization processes (Gilbert \& Wiesel, 1992), where subthreshold influences from neurons with neighboring RFs quickly became suprathreshold when the ascending input was eliminated. Here, neurons with RFs "along" the illusory contour were also lacking ascending input, but instead, may have received excitation via lateral connections from neurons with a collinear orientation preference, which were bottom-up stimulated by the edges of the inducing stimuli (Figure 3A). This process of recruitment of neurons coding the illusory contour is thought to underlie the observed increase in the BOLD response from pre- to posttraining.

\section{Top-Down Contributions}

In addition to contextual interactions that are mediated by horizontal interactions, feedback influences must also be considered (Lee, 2003). fMRI responses to illusory surfaces have been observed in the human LOC (Stanley \& Rubin, 2003; Mendola et al., 1999), previously assumed to reflect the source of feedback influences guiding the illusory contour completion process in the primary visual cortex via the definition of a "salient region." In the learning group, a decrease in LOC activation was observed between the pre- and the posttraining block. Initially, the cascade mechanism, which we propose to form an IC representation in the primary visual cortex, may have been supported by feedback input from the LOC. As a consequence of training, however, top-down influences may have been diminished in favor of an autonomic, task-specific representation in the primary visual cortex. This is supported by the observed acuity losses following the change in the retinotopic, and therefore, cortical position in the transfer test. If the learning effect had been implemented exclusively in the LOC, the position change would have had no effect, due to the size invariance of the neural response of LOC neurons. We conclude that even relatively short-term, within-session training induced durable behavioral changes, which can be attributed to modifications in the primary visual cortex.

\section{METHODS}

\section{Subjects and Task}

Twenty-four right-handed subjects (12 women) with a mean age of 26 years (age range 21-37 years) gave informed consent to participate in the experiment according to the guidelines of the Max-Planck Institute. The fMRI procedures were approved by the ethics review board of the University of Leipzig.

Subjects were trained in a curvature discrimination task, adapted from Rubin and colleagues (1997). The task involved the classification of shapes as concave or convex on the basis of the perceived curvature in the bounding illusory contours. Stimuli were presented on a black background and consisted of four white segments, the "pacmen" inducers, at the corners of an imaginary square. By manipulating the angle $\alpha$ of the inducer opening, the resulting Kanizsa-like illusory figure can be classified as convex ("fat") for $\alpha>90^{\circ}$ and as concave ("thin") for $\alpha<90^{\circ}$ (Figure 1A). In contrast to earlier studies, in which rectangular openings were rotated to create a curvature impression, here only the side edges were curved, whereas the upper and lower edges were straight lines. Stimulus parameters were determined on the basis of four pilot experiments ( $n=6$ each), in which we tested several SRs, defined as the ratio between the luminance-defined part of an illusory contour and its total side length $(\mathrm{SR}=0.2,0.25,0.225)$, and different segment angles for the training block. Finally, in order to make the task demanding enough to leave room for improvements, we employed an SR of 0.225. Pacmen diameter was $3.24^{\circ}$ visual angle and the side length of the Kanizsa figure was $14.3^{\circ}$ corresponding to $4.9 \mathrm{~cm}$ and $22 \mathrm{~cm}$ at a viewing distance of $85 \mathrm{~cm}$. Stimuli were displayed on a backprojection screen, mounted in the bore of the magnet behind the participant's head, using a liquid crystal display (LCD) projector. Participants viewed the screen by wearing mirrored glasses. A trial started with a fixation period of $1000 \mathrm{msec}$ (Figure 2). Then, a Kanizsa figure was presented for $100 \mathrm{msec}$, which was followed, after $66 \mathrm{msec}$, by a masking stimulus consisting of four asterisks (Figure 2) in the corner positions of the inducers, which lasted for 
250 msec. Participants were asked to steadily fixate the central cross whenever it was presented, and considering the presentation time of $100 \mathrm{msec}$ for the Kanizsa figures, the systematic occurrence of eye movements is rather unlikely. The subjects indicated whether the stimulus was perceived as thin or fat by pressing a button with their index or middle finger, respectively. Trial duration was fixed at $5 \mathrm{sec}$. The experiments were performed using Presentation ${ }^{(\mathrm{II})}$ software (Version 0.61, www.neurobs.com).

In a method-of-constant-stimuli procedure eight different $\alpha$ values were presented, consisting of more difficult discrimination conditions $\left(90^{\circ} \pm 0.5^{\circ}, 1^{\circ}, 2^{\circ}, 3^{\circ}\right)$ for the test blocks, and easier conditions $\left(90^{\circ} \pm 3^{\circ}, 4^{\circ}\right.$, $5^{\circ}, 6^{\circ}$ ) for the intervening training block. Within a block, each of the four fat and thin stimuli were presented 10 times together with 16 null events (e.g., fixationcross-only trials with a duration of $5 \mathrm{sec}$ ). Each of the two test blocks, as well as the training block, were performed in the MR scanner.

We were able to retest 15 participants ( $n=7$ learners) in a behavioral follow-up session outside the MR scanner, performed, on average, 10 months after the initial testing. The retain test involved two blocks equivalent to the initial test blocks except for the null events, which were missing in the retest. In the transfer test, two blocks of 80 trials each were performed with the same level of difficulty as in the test blocks $\left(\alpha=90^{\circ} \pm 0.5^{\circ}, 1^{\circ}\right.$, $2^{\circ}, 3^{\circ}$ ), but with inducer stimuli that were half the size of the original $\left(1.12^{\circ}\right)$, and that were presented at half of the eccentricity $\left(3.57^{\circ}\right.$ to the left and to the right, and upper and lower fixation).

\section{Estimating Psychometric Functions}

Together, $\alpha$ and $\beta$ determine the horizontal displacement of the curve, whereby $\beta$ is related to the slope of the curve and $\alpha$ describes for which value of $x$ the function takes the value of 0.5 , because setting $\alpha=x$ :

$$
f(\alpha)=\frac{1}{1+e^{-\frac{\alpha-\alpha}{\beta}}}=\frac{1}{1+e^{0}}=\frac{1}{2}
$$

The guessing rate $\gamma$ and the lapsing rate $\lambda$ determine the lower and upper performance bounds of predicted performance. The value of $\gamma$ corresponds to predicted performance in the absence of stimulation and is fixed at the reciprocal of the number of alternatives per trial producing a value for $\gamma$ of $0.5 .(1-\lambda)$ is the predicted performance for an arbitrarily large stimulus, and because we assume that observers do not make stimulusindependent errors at a large rate, $\lambda$ was allowed to vary maximally between 0 and 0.05 .

Variance estimates were obtained using a bootstrap method, the bias-corrected and accelerated (BCa) boot- strap implemented by Psignifit, based on 2000 simulations (Wichmann \& Hill, 2001b).

\section{fMRI Data Acquisition and Analysis}

The fMRI data were acquired at a field strength of $3 \mathrm{~T}$ (Medspec 30/100, Bruker, Ettlingen, Germany) using a single-shot, gradient-recalled, echo-planar imaging (EPI) sequence with a TR $=2000 \mathrm{msec}$, TE $=30 \mathrm{msec}$, and a flip angle of $90^{\circ}$. The in-plane resolution was $3 \times 3 \mathrm{~mm}$ with an FOV of $19.2 \mathrm{~cm}$. Twenty-two slices were acquired with $4 \mathrm{~mm}$ slice thickness and $1 \mathrm{~mm}$ interslice gap. The slices were oriented horizontally with the eighth most inferior slice in the AC-PC plane. Prior to the functional runs, corresponding sets of 22 anatomical MDEFT and T1-EPI slices were acquired. In the first functional scan we measured brain activity underlying perceptual learning and in the second functional scan we performed a retinotopic mapping to functionally localize the representation of the learning stimuli in the early visual areas.

The fMRI time series were analyzed with the software package LIPSIA (Leipzig Image Processing and Statistical Inference Algorithms; Lohmann et al., 2001). Initially, data were corrected for movement artifacts and time differences in slice acquisition were corrected by sinc interpolation. The experimental data, but not the data from the localizer scan, were smoothed with a Gaussian filter with full width at half maximum (FWHM) $=7 \mathrm{~mm}$. A temporal high-pass filter with a cutoff frequency of $1 / 40 \mathrm{~Hz}$ was used to remove low-frequency drifts.

The functional datasets were aligned with the Talairach space (Talairach \& Tournoux, 1988) in three steps. A matrix describing a rigid, affine linear transformation with three translational and three rotational parameters was computed that registered the 2-D anatomical MDEFT images with individual high-resolution 3-D datasets. The transformation matrix was multiplied by linear scaling factors to adjust for deviations from standard size and applied for registering the functional data.

The statistical evaluation was based on the general linear model for serially autocorrelated observations (Worsley \& Friston, 1995). For each individual subject, statistical parametric maps (SPM) were generated. The design matrix for event-related analysis was created using a synthetic model of the hemodynamic response function (Josephs \& Henson, 1999), which includes an estimate of temporal autocorrelation.

\section{A Priori Regions of Interest}

The pre- and postlearning fMRI time series were analyzed specifically in those voxels along the calcarine fissure that were functionally identified as the site of the representation of the illusory contours within V1. For this purpose, real contours of $0.23^{\circ}$ thickness, and 
with length identical to the illusory part of the edges of the Kanizsa square, were presented in alternation at the position of the left, right, upper, or lower edges of the Kanizsa stimulus, or simultaneously at all four edges. For eight subjects, left and right contours were presented simultaneously. (Stimuli turned on and off every $125 \mathrm{msec}$ - contrast reversal frequency of $8 \mathrm{~Hz}$ ). Horizontal and vertical meridian mappings were performed, presenting perspective-scaled checkerboard wedges to account for cortical magnification, centered in two $60^{\circ}$ arcs around the horizontal and vertical midline, and extending to $9.1^{\circ}$ eccentricity, with a contrast-reversal frequency of $8 \mathrm{~Hz}$. Five stimulus cycles were run with six blocks of 10-sec periods for each stimulus. In one such period, stimuli flickered (or contrast-reversed) for $2 \mathrm{sec}$ and a blank screen was shown for the remaining $8 \mathrm{sec}$. Event-related BOLD responses were averaged timelocked to stimulus onset. ROIs were determined in the left and right visual cortex of all individual brains (Table 1, Figure 4). We selected the voxels with maximal activation in response to contralaterally presented real contours along the banks (typically at or near the fundus) of the calcarine fissure. To ensure that the activation marked the contour representation in V1, we also analyzed the activation elicited by the vertical checkerboard wedges in the localizer scan (which mark the dorsal and ventral representation of the vertical meridian, and thus, the borders between V1 and V2v and V2d; Sereno et al., 1995) and accepted only contour representations which, in the sagittal image, lay along the banks of the calcarine between the dorsal and ventral representation of the vertical meridian.

From these voxels, we then extracted the preprocessed (see above) fMRI signal evoked by Kanizsa-type stimuli (i.e., by "illusory contours") in all (correct and incorrect) trials. This was performed for two reasons: First, the estimation of psychometric functions and the derivation of perceptual thresholds (i.e., the behavioral measure used to discriminate between learners and nonlearners) relied on both correct and false answers, as well. Secondly, because there have been less correct responses in the first than in the second test block, a reduced BOLD response in the first block may be attributed to a problem of statistical power resulting from the lower number of observations. We also pooled data across rotation angles, because at an in-plane resolution of $3 \mathrm{~mm}$ we would not expect to be able to reliably differentiate between activity patterns evoked by convex and concave stimuli. Even at the point of maximum curvature amidst the pacmen inducers and for the maximum inducer opening of $87^{\circ}$ or $93^{\circ}$, the distance between convex and concave contours was only $0.86^{\circ}$ visual angle. Trial averages were computed over a time window of $20 \mathrm{sec}$ poststimulus onset. The average signal time courses in response to null events were subtracted from the corresponding signal time courses in the experimental conditions and the differences were ex- pressed as percent signal change from the null-event signal. The subtraction of null events from experimental trials was performed separately for each block to remove potential artifacts caused by slow signal drifts. Variance estimates were calculated using the jackknife bootstrapping technique (Ruge, Brass, Lohmann, \& von Cramon, 2003; Efron, 1977), in which the original data were resampled by computing $n$ new elements, each of which represents the average of $(n-1)$ fMRI time series of the original sample. The basic principle of this method is that if all participants show approximately the same effect, then the resampled values should be quite similar for whichever subject is omitted. If the participants show different effects, however, then the results should fluctuate substantially depending on which subject is left out. Adjusted formula were devised to compute jackknifed estimates of the standard error of the mean, accounting for reductions in variance due to resampling as well as for the standard error of the mean of differences used to compute the repeated measures $t$-statistic:

$$
S E_{\mathrm{DIFF}}=\sqrt{\frac{N-1}{N} \sum_{i_{-1}=1}^{N}\left(d(i)_{-1}-\bar{D}\right)^{2}}
$$

We computed multiple $t$ tests over timesteps to test for differences between the BOLD signal in response to illusory contours in the pretraining versus posttraining block. In order to correct for multiple comparisons, the FDR procedure was applied on the resulting $p$ values for all timesteps using the procedures introduced by Benjamini and Hochberg (1995) (see also Genovese, Lazar, \& Nichols, 2002). These procedures control for the FDR, which is the expected proportion of erroneously rejected among all rejected hypotheses. The value of $q$ specifying the maximum FDR was set to 0.05 , indicating that in the number of tests deemed significant in a given experiment, there were (on average) no more than 5\% errors.

\section{Whole-brain Analysis}

To examine whether there were further differences in the average neural activity of "learners" and "nonlearners," in addition to those predicted for the primary visual cortex, we computed two sets of individual contrast images, namely, differences in parameter estimates ( $\beta$ values) between pre- and posttraining adjusted for the corresponding null events ( $\left[\mathrm{BOLD}_{\text {pretraining }}-\right.$ BOLD $\left.\left.D_{\text {pre_null }}\right]-\left[\mathrm{BOLD}_{\text {posttraining }}-\mathrm{BOLD}_{\text {post_null }}\right]\right)$ for the two subject groups. In a random effects analysis, the two sets of contrast images were compared voxelwise using a two-sample $t$ test. The resulting $t$ values were transformed so that the resulting image contained $Z$-scores indicating the degree of significance of the 
group difference. To minimize the probability of false positives (type I error), only voxels with a $Z$-score $>3.09$ $(p<.001$, uncorrected) in a cluster with a volume of $>225 \mathrm{~mm}^{3}$ (eight contiguous voxels) were considered.

\section{Acknowledgments}

M. M. was supported by the Gertrud Reemtsma Foundation. S. P. was in part supported by the European Union (Grant no. QLG-CT-2002-SISS2). The fMRI experiments were carried out at the Max Planck Institute of Human Cognitive and Brain Sciences.

We thank two anonymous reviewers for their constructive comments. We thank Daniel Lucey for proofreading this manuscript.

The data reported in this experiment have been deposited with the fMRI Data Center archive www.fmridc.org). The accession number is 2-2005-118TB.

Reprint requests should be sent to Marianne Maertens, Institute of Psychology II, Department of Experimental Psychology, Otto-von-Guericke University, Universitätsplatz 2, D-39106 Magdeburg, Germany, or via e-mail: marianne.maertens@nat. uni-magdeburg.de.

\section{Note}

1. In two participants, even at a liberal threshold of $z=1.64$, $p=.05$, activation changes in response to contralaterally presented checkerboard bars could not be identified in V1.

\section{REFERENCES}

Ahissar, M., \& Hochstein, S. (1997). Task difficulty and the specificity of perceptual learning. Nature, 387, 401-406.

Ahissar, M., \& Hochstein, S. (2004). The reverse hierarchy theory of visual perceptual learning. Trends in Cognitive Sciences, 8, 457-464.

Angelucci, A., Levitt, J. B., Walton, E. J., Hupe, J. M., Bullier, J., \& Lund, J. S. (2002). Circuits for local and global signal integration in primary visual cortex. Journal of Neuroscience, 22, 8633-8646.

Beauchamp, M. S., Cox, R. W., \& DeYoe, E. A. (1997). Graded effects of spatial and featural attention on human area MT and associated motion processing areas. Journal of Neurophysiology, 78, 516-520.

Benjamini, Y., \& Hochberg, Y. (1995). Controlling the false discovery rate: A practical and powerful approach to multiple testing. Journal of Royal Statistical Society, Series B, 57, 289-300.

Bosking, W. H., Zhang, Y., Schofield, B., \& Fitzpatrick, D. (1997). Orientation selectivity and the arrangement of horizontal connections in tree shrew striate cortex. Journal of Neuroscience, 17, 2112-2127.

Brefczynski, J. A., \& DeYoe, E. A. (1999). A physiological correlate of the "spotlight" of visual attention. Nature Neuroscience, 2, 370-374.

Büchel, C., Josephs, O., Rees, G., Turner, R., Frith, C. D., \& Friston, K. J. (1998). The functional anatomy of attention to visual motion. A functional MRI study. Brain, 121, 1281-1294.

Corbetta, M., Akbudak, E., Conturo, T. E., Snyder, A. Z., Ollinger, J. M., Drury, H. A., Linenweber, M. R., Petersen, S. E., Raichle, M. E., Van Essen, D. C., \& Shulman, G. L.
(1998). A common network of functional areas for attention and eye movements. Neuron, 21, 761-773.

Efron, B. (1977). Bootstrap methods: Another look at the jackknife. Annals of Statistics, 7, 1-26.

Fahle, M., \& Poggio, T. (2002). Perceptual learning. Cambridge: MIT Press.

Fahle, M., Edelman, S., \& Poggio, T. (1995). Fast perceptual learning in hyperacuity. Vision Research, 35, 3003-3013.

Ffytche, D. H., \& Zeki, S. (1996). Brain activity related to the perception of illusory contours. Neuroimage, 3, 104-108.

Fiorentini, A., \& Berardi, N. (1980). Perceptual learning specific for orientation and spatial frequency. Nature, 287, 43-44.

Gandhi, S. P., Heeger, D. J., \& Boynton, G. M. (1999). Spatial attention affects brain activity in human primary visual cortex. Proceedings of the National Academy of Sciences, U.S.A., 96, 3314-3319.

Genovese, C. R., Lazar, N. A., \& Nichols, T. (2002). Thresholding of statistical maps in functional neuroimaging using the false discovery rate. Neuroimage, 15, 870-878.

Gilbert, C. D., Sigman, M., \& Crist, R. E. (2001). The neural basis of perceptual learning. Neuron, 31, 681-697.

Gilbert, C. D., \& Wiesel, T. N. (1992). Receptive field dynamics in adult primary visual cortex. Nature, 356, 150-152.

Gitelman, D. R., Nobre, A. C., Parrish, T. B., LaBar, K. S., Kim, Y. H., Meyer, J. R., \& Mesulam, M. (1999). A large-scale distributed network for covert spatial attention: Further anatomical delineation based on stringent behavioural and cognitive controls. Brain, 122, 1093-1106.

Grill-Spector, K., Kushnir, T., Hendler, T., Edelman, S., Itzchak, Y., \& Malach, R. (1998). A sequence of object-processing stages revealed by fMRI in the human occipital lobe. Human Brain Mapping, 6, 316-328.

Grosof, D. H., Shapley, R. M., \& Hawken, M. J. (1993). Macaque V1 neurons can signal "illusory" contours. Nature, 365, 550-552.

Heeger, D. J., \& Ress, D. (2002). What does fMRI tell us about neuronal activity? Nature Reviews Neuroscience, 3, 142-151.

Hirsch, J., DeLaPaz, R. L., Relkin, N. R., Victor, J., Kim, K., Li, T., Borden, P., Rubin, N., \& Shapley, R. (1995). Illusory contours activate specific regions in human visual cortex: Evidence from functional magnetic resonance imaging. Proceedings of the National Academy of Sciences, U.S.A., 92, 6469-6473.

Hochstein, S., \& Ahissar, M. (2002). View from the top: Hierarchies and reverse hierarchies in the visual system. Neuron, 36, 791-804.

Hubel, D. H., \& Wiesel, T. N. (1977). Functional architecture of macaque monkey visual cortex. Proceedings of the Royal Society of London, Series B, Biological Sciences, 198, 1-59.

Josephs, O., \& Henson, R. N. (1999). Event-related functional magnetic resonance imaging: Modelling, inference and optimization. Philosophical Transactions of the Royal Society of London, Series B, Biological Sciences, 354, 1215-1228.

Karni, A., \& Sagi, D. (1991). Where practice makes perfect in texture discrimination: Evidence for primary visual cortex plasticity. Proceedings of the National Academy of Sciences, U.S.A., 88, 4966-4970.

Kastner, S., De Weerd, P., Desimone, R., \& Ungerleider, L. G. (1998). Mechanisms of directed attention in the human extrastriate cortex as revealed by functional MRI. Science, 282, 108-111.

Kastner, S., De Weerd, P., Pinsk, M. A., Elizondo, M. I., Desimone, R., \& Ungerleider, L. G. (2001). Modulation of sensory suppression: Implications for receptive field sizes in the human visual cortex. Journal of Neurophysiology, 86, 1398-1411. 
Larsson, J., Amunts, K., Gulyas, B., Malikovic, A., Zilles, K., \& Roland, P. E. (1999). Neuronal correlates of real and illusory contour perception: Functional anatomy with PET. European Journal of Neuroscience, 11, 4024-4036.

Lee, T. S., \& Nguyen, M. (2001). Dynamics of subjective contour formation in the early visual cortex. Proceedings of the National Academy of Sciences, U.S.A., 98, 1907-1911.

Lee, T. S. (2003). Computations in the early visual cortex. Journal of Physiology (Paris), 97, 121-139.

Lobley, K., \& Walsh, V. (1998). Perceptual learning in visual conjunction search. Perception, 27, 1245-1255.

Lohmann, G., Muller, K., Bosch, V., Mentzel, H., Hessler, S., Chen, L., Zysset, S., \& von Cramon, D. Y. (2001). LIPSIA-A new software system for the evaluation of functional magnetic resonance images of the human brain. Computerized Medical Imaging and Graphics, 25, 449-457.

Martinez, A., Anllo-Vento, L., Sereno, M. I., Frank, L. R., Buxton, R. B., Dubowitz, D. J., Wong, E. C., Hinrichs, H., Heinze, H. J., \& Hillyard, S. A. (1999). Involvement of striate and extrastriate visual cortical areas in spatial attention. Nature Neuroscience, 2, 364-369.

Mendola, J. D., Dale, A. M., Fischl, B., Liu, A. K., \& Tootell, R. B. (1999). The representation of illusory and real contours in human cortical visual areas revealed by functional magnetic resonance imaging. Journal of Neuroscience, 19, $8560-8572$.

Mollon, J. D., \& Danilova, M. V. (1996). Three remarks on perceptual learning. Spatial Vision, 10, 51-58.

O'Connor, D. H., Fukui, M. M., Pinsk, M. A., \& Kastner, S. (2002). Attention modulates responses in the human lateral geniculate nucleus. Nature Neuroscience, 5, 1203-1209.

O'Craven, K. M., Rosen, B. R., Kwong, K. K., Treisman, A., \& Savoy, R. L. (1997). Voluntary attention modulates fMRI activity in human MT-MST. Neuron, 18, 591-598.

Pillow, J., \& Rubin, N. (2002). Perceptual completion across the vertical meridian and the role of early visual cortex. Neuron, 33, 805-813.

Pollmann, S., Weidner, R., Müller, H. J., \& von Cramon, D. Y. (in press). Neural correlates of visual dimension weighting. Visual Cognition.

Pollmann, S., \& von Cramon, D. Y. (2000). Object working memory and visuospatial processing: Functional neuroanatomy analyzed by event-related fMRI.

Experimental Brain Research, 133, 12-22.

Pollmann, S., Weidner, R., Muller, H. J., \& von Cramon, D. Y. (2000). A fronto-posterior network involved in visual dimension changes. Journal of Cognitive Neuroscience, 12, 480-494.

Ramsden, B. M., Hung, C. P., \& Roe, A. W. (2001). Real and illusory contour processing in area V1 of the primate: A cortical balancing act. Cerebral Cortex, 11, 648-665.

Ringach, D. L., \& Shapley, R. (1996). Spatial and temporal properties of illusory contours and amodal boundary completion. Vision Research, 36, 3037-3050.

Ritzl, A., Marshall, J. C., Weiss, P. H., Zafiris, O., Shah, N. J., Zilles, K., \& Fink, G. R. (2003). Functional anatomy and differential time courses of neural processing for explicit, inferred, and illusory contours. An event-related fMRI study. Neuroimage, 19, 1567-1577.
Rubin, N., Nakayama, K., \& Shapley, R. (1997). Abrupt learning and retinal size specificity in illusory contour perception. Current Biology, 7, 461-467.

Ruge, H., Brass, M., Lohmann, G., \& von Cramon, D. Y. (2003). Event-related analysis for event types of fixed order and restricted spacing by temporal quantification of trialaveraged fMRI time courses. Journal of Magnetic Resonance Imaging, 18, 599-607.

Schiltz, C., Bodart, J. M., Dubois, S., Dejardin, S., Michel, C., Roucoux, A., Crommelinck, M., \& Orban, G. A. (1999). Neuronal mechanisms of perceptual learning: Changes in human brain activity with training in orientation discrimination. Neuroimage, 9, 46-62.

Schwartz, S., Maquet, P., \& Frith, C. (2002). Neural correlates of perceptual learning: A functional MRI study of visual texture discrimination. Proceedings of the National Academy of Sciences, U.S.A., 99, 17137-17142.

Sereno, M. I., Dale, A. M., Reppas, J. B., Kwong, K. K,, Belliveau, J. W., Brady, T. J., Rosen, B. R., \& Tootell, R. B. (1995). Borders of multiple visual areas in humans revealed by functional magnetic resonance imaging. Science, 268, 889-893.

Sheth, B. R., Sharma, J., Rao, S. C., \& Sur, M. (1996). Orientation maps of subjective contours in visual cortex. Science, 274, 2110-2115.

Shipley, T. S., \& Kellman, P. J. (1992). Strength of visual interpolation depends on the ratio of physically specified to total edge length. Perception \& Psychophysics, 52, 97-106.

Somers, D. C., Dale, A. M., Seiffert, A. E., \& Tootell, R. B. (1999). Functional MRI reveals spatially specific attentional modulation in human primary visual cortex. Proceedings of the National Academy of Sciences, U.S.A., 96, 1663-1668.

Stanley, D. A., \& Rubin, N. (2003). fMRI activation in response to illusory contours and salient regions in the human lateral occipital complex. Neuron, 37, 323-331.

Strasburger, H. (2001). Converting between measures of slope of the psychometric function. Perception \& Psychophysics, 63, 1348-1355.

Talairach, J., \& Tournoux, P. (1988). A co-planar stereotactic atlas of the buman brain. Stuttgart: Thieme.

Tootell, R. B., Hadjikhani, N., Hall, E. K., Marrett, S., Vanduffel, W., Vaughan, J. T., \& Dale, A. M. (1998). The retinotopy of visual spatial attention. Neuron, 21, 1409-1422.

Toth, L. J., Rao, S. C., Kim, D. S., Somers, D., \& Sur, M. (1996). Subthreshold facilitation and suppression in primary visual cortex revealed by intrinsic signal imaging. Proceedings of the National Academy of Sciences, U.S.A., 93, 9869-9874.

von der Heydt, R., Peterhans, E., \& Baumgartner, G. (1984). Illusory contours and cortical neuron responses. Science, 224, 1260-1262.

Watanabe, T., Nanez, J. E., \& Sasaki, Y. (2001). Perceptual learning without perception. Nature, 413, 844-848.

Wichmann, F. A., \& Hill, N. J. (2001a). The psychometric function: I. Fitting, sampling and goodness-of-fit. Perception E Psychophysics, 63, 1293-1313.

Wichmann, F. A., \& Hill, N. J. (2001b). The psychometric function: II. Bootstrap-based confidence intervals and sampling. Perception \& Psychophysics, 63, 1314-1329.

Worsley, K. J., \& Friston, K. J. (1995). Analysis of fMRI timeseries revisited-again. Neuroimage, 2, 173-181. 